\title{
Remdesivir for the Treatment of COVID- 19: A Need for Combined In Vivo and In Vitro Studies to Evaluate the Efficacy
}

Journal of Pharmacy Practice 202I, Vol. 34(3) 343-346 (C) The Author(s) 2021 Article reuse guidelines: sagepub.com/journals-permissions DOI: | 0.1 I77/0897| 9002199700 | journals.sagepub.com/home/jpp (S)SAGE

\author{
Muhammad Naveed, PharmD, MPhil' $\odot$, Shahab Uddin, Bs, MPhil' \\ Muhammad Khalid Khan, PharmD, MPhil ${ }^{3}$, \\ and Zakir Khan, PharmD, MPhil ${ }^{4}(\mathbb{D}$
}

The SARS-CoV-2 is a highly infective and transmittable and ever since it has spread worldwide quickly and became a global public health challenge. ${ }^{1,2}$ Right now, due to lack of a vaccine or specific antiviral treatment for human and animal coronaviruses (CoVs), urgent identification of drug treatment for the response to the COVID-19 outbreak is the utmost requirement. $^{3}$ Effective antiviral therapy is important to tackle the COVID-19 pandemic. Up to date, several antivirals, previously developed or used as treatments for SARS (Severe Acute Respiratory Syndrome), MERS (Middle East Respiratory Syndrome), human immunodeficiency virus (HIV), and malaria, have been investigated against COVID-19 and some have moved into clinical trials conducted around the globe. ${ }^{4}$ Some of them have been achieved promising results so far. ${ }^{5}$ At least 23 studies of remdesivir are presently recorded on various trial registers, proposing to study 23,500 patients, but fewer than a quarter are double-blind, and some are uncontrolled observational studies. ${ }^{6}$ Additional information on some of the ongoing remdesivir clinical studies ${ }^{7}$ is presented in Table 1 . Despite of the fact, remdesivir is being considered the most effective potential agent against COVID-19 based on preliminary in vitro and clinical studies, it still requires an elaborative research study combining quantitatively large direct clinical trials and in vitro studies to approve its efficacy and safety. This study aims to highlight the potential of remdesivir in antiCOVID-19 research to help inform clinicians considering the use of remdesivir.

Remdesivir, a prodrug of a nucleotide analogue that prevents the action of viral RNA polymerases. The original pioneer of remdesivir "Gilead Sciences" developed the drug to combat Ebola and related viruses by blocking the RNAdependent RNA polymerase (RdRp) of pathogens. ${ }^{8}$ During the past outbreaks that occurred due to members of few other virus families containing Filoviruses (Ebola) and CoVs (MERS/ SARS), the antiviral remdesivir has considered to possess broad-spectrum activity against these viruses. ${ }^{9}$ It has also been reported from several studies that the drug holds therapeutic efficacy and prophylactic activity in many nonclinical models of the above CoVs. ${ }^{10}$ A report published in the journal of NATURE biotechnology stated that the broad-spectrum antiviral "remdesivir has quite a high potency against all different CoVs and therefore it is selected as one of the prime and suitable drug candidates to start being tested."11 World Health Organization (WHO) in the mid of March had launched an international level mega trial of the 4 most auspicious treatments against $\mathrm{CoV}$ including remdesivir on top of the list with higher potency and efficacy. According to their study, the SARS-CoV-2 is giving this compound a second chance to shine. A virtual meeting held on 2nd April 2020 among members of EMA's (Human Medicines Committee) provided recommendations on concerned usage of remdesivir as an efficient antiviral for COVID-19 medication. ${ }^{12}$

Recently, when there is a race to find a potent drug candidate, remdesivir was also tested in vitro and results have presented that it has prominent activity against SARS-CoV-2 and chosen as a strong candidate (Table 2). In vitro remdesivir has found effective at EC90 of $1.76 \mu \mathrm{M}$ against COVID-19 in one study. In this study, the antiviral efficacy of remdesivir was evaluated against a clinical isolate of SARS-CoV-2 in vitro in Vero E6 cells and observed to potently blocked virus infection at a low-micromolar concentration $($ EC50 $=0.77 \mu \mathrm{M}) .^{13}$ According to Agostini et al., remdesivir potently inhibits human and zoonotic CoVs in vitro and in a SARS-CoV mouse model. They demonstrated that remdesivir inhibits CoVs (MERS and SARS) early after infection by interfering with

\footnotetext{
'Department of Pharmacology and Pharmacotherapy, Faculty of Medicine, University of Szeged, Szeged, Hungary

${ }^{2}$ Department of Microbiology, School of Life Sciences, Lanzhou University, Lanzhou, China

${ }^{3}$ Department of Pharmaceutics, Faculty of Pharmacy, Gomal University, DI Khan, Pakistan

${ }^{4}$ Tıbbi Farmakoloji Anabilim Dalı, Tıp Fakultesi, Cukurova Universitesi, Sarıcam, Adana, Turkey
}

Corresponding Author:

Muhammad Naveed, Department of Pharmacology and Pharmacotherapy, Faculty of Medicine, University of Szeged, Szeged, Hungary.

Email: muhammadnaveedkhan0I@gmail.com 
Table I. Ongoing Remdesivir Clinical Studies.

Ongoing remdesivir clinical studies against COVID-19

\begin{tabular}{|c|c|c|c|c|}
\hline Type of study & Study phase & Recruitment status & Estimated primary completion date & Identifier \\
\hline - Interventional & - Early Phase I & - Recruiting & - November 30, 2020 & - NCT0456023I \\
\hline - Interventional & - Phase 3 & - Recruiting & - December 2020 & - NCT0450I952 \\
\hline - Interventional & - Phase 2 Phase 3 & - Recruiting & - February 2021 & - NCT0443 I 453 \\
\hline - Interventional & - Phase 3 & - Recruiting & - March 2023 & - NCT043I5948 \\
\hline
\end{tabular}

Table 2. Summary of Remdesivir Findings (In Vitro) Reported in Selected Articles.

In vitro effectiveness of remdesivir against CoVs and COVID-19

\begin{tabular}{llll}
\hline Type of study & Target & Outcomes & References \\
\hline- In vitro (using Vero E6 cells) & - SARS-CoV-2 & - EC50 $=0.77 \mu$ M against COVID-19 & 13 \\
- In vitro & - MERS and SARS & - EC50 $=0.07 \& 0.069 \mu$ M against MERS and SARS-CoVs, respectively & 14 \\
- In vitro & - RNA viruses & - Potent inhibition of RNA viruses in cell based assays & 8 \\
- Opinion & - COVID-19 & - Appropriate potential biocontainment capability against COVID-I9 & 15 \\
- In silico & - SARS-CoV-2 & - Suggested as a potent drug against COVID-19 & 9 \\
\hline
\end{tabular}

Abbreviations: CoVs (coronaviruses), COVID-19 (2019 coronavirus disease), SARS-CoV-2 (severe acute respiratory syndrome coronavirus 2), EC50 (half maximal effective concentration), MERS (Middle East respiratory syndrome), SARS (severe acute respiratory syndrome), RNA (ribonucleic acid).

viral RNA replication at $\mathrm{EC}_{50}$ value of $0.07 \mu \mathrm{M}$ and $0.069 \mu \mathrm{M}$, respectively. ${ }^{14}$ Moreover, Gordon et al., demonstrated that remdesivir potently inhibits RNA viruses in cell-based assays. ${ }^{8}$ In addition, Guangdi Li \& De Clercq also claimed that remdesivir has an appropriate potential biocontainment capability against COVID-19. ${ }^{15}$ Likewise, an in silico model built by sequence analysis, modeling and, docking suggested the effectiveness of remdesivir as a potent drug against the newly developed COVID-19. . Furthermore, in an animal study "rhesus macaque model of MERS-CoV infection," prophylactic remdesivir treatment prevented MERS-CoV-induced clinical disease and lung lesions in rhesus macaques inoculated with MERS$\mathrm{CoV}$, and strongly inhibited MERS-CoV replication in respiratory tissues (Table 3). Since SARS-CoV-2 is mostly related to these $\mathrm{CoVs}$, it opens the gateway and could be considered for implementation in clinical trials against SARS-CoV-2. ${ }^{16}$

Presently, there have been successful cases of remdesivir in treating COVID-19. The first case of COVID-19 reported in the US was a young man in Washington. He was administered with antiviral remdesivir upon continuous aggravation of the clinical symptoms on the 6th day of admission, and began to continue intravenous (I.V) remdesivir to the patient on the following day. On the 8th day of admission, the patient's clinical symptoms were improved, and the oxygen saturation increased to $94 \% .{ }^{17}$ Moreover, a study entitled "Compassionate Use of Remdesivir for Patients with Severe COVID-19" published by $\mathrm{J}$. Grein et al., reported a total of 53 infection cases of COVID-19 that were hospitalized in more than 20 hospitals of several affected countries, were treated with remdesivir. The median duration of symptoms before the initiation of remdesivir treatment was 12 days. These patients (who had an oxygen saturation of $94 \%$ or less while they were breathing ambient air or who were receiving oxygen support) received the drug between January 25 and March 7, 2020, in hospitals around the world: 22 in Europe, 22 in the USA and, 9 in Japan. A 10-day treatment course with $200 \mathrm{mg}$ loading dose on day 1 and $100 \mathrm{mg}$ daily I.V up to remaining 9 days was followed and the overall probability of improvement in the category of oxygen support of patients by 18 days was $68 \% .23 \%$ patients in this study experienced serious adverse events such as multiple-organ-dysfunction syndrome, septic shock, acute kidney injury, and hypotension. ${ }^{18}$

Conferring to the Centers for Disease Control and Prevention (CDC), until March 2020, one investigational agent (remdesivir) was in use in the USA. ${ }^{4}$ Recently, an emergency approval has granted by the U.S. Food and Drug Administration (USFDA) to use remdesivir against COVID-19 in more hospitals around the United States, ${ }^{21}$ making it the first authorized therapy for the virus. USFDA's approval was based on trial results which revealed that the drug reduced the recovery time to 5 days from 10 days for moderate patients (who have evidence of lower respiratory disease by clinical assessment or imaging and a saturation of oxygen $\left(\mathrm{SpO}_{2}\right) \geq 94 \%$ on room air at sea level). Similarly, Japan also approved remdesivir for the treatment of severe COVID-19 patients, under an exceptional approval pathway based on clinical data from the U.S. National 
Table 3. Summary of Remdesivir Findings (In Vivo) Reported in Selected Articles.

In vivo effectiveness of remdesivir against CoVs and COVID-19

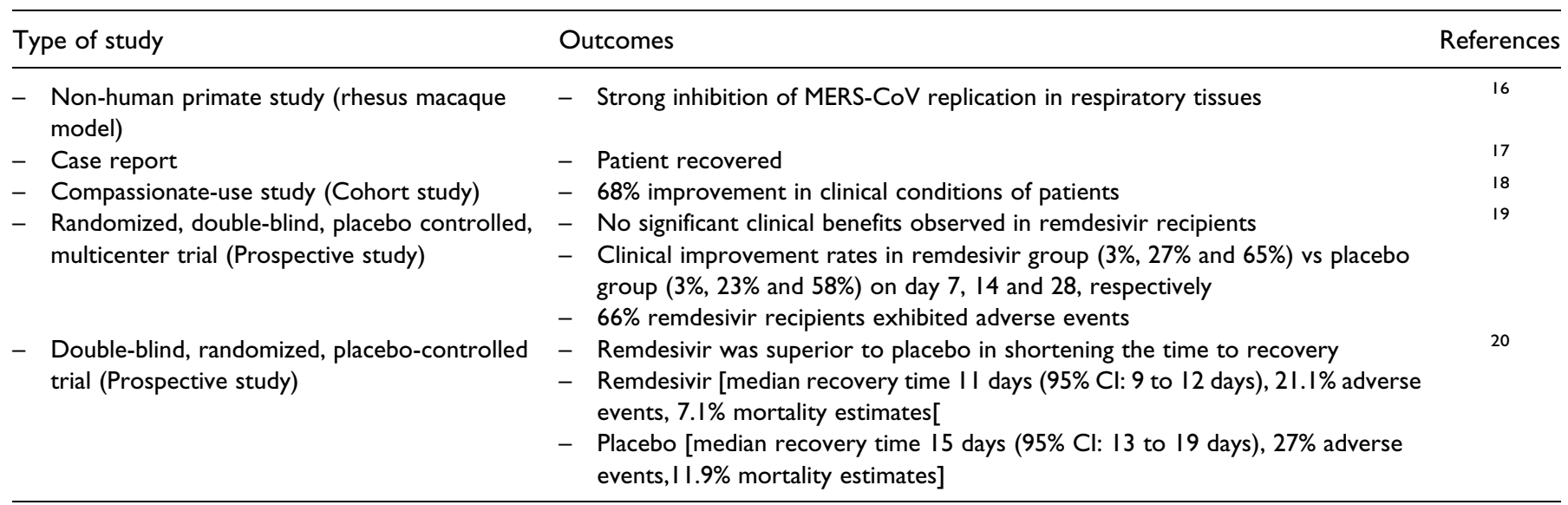

Abbreviations: CoVs (coronaviruses), COVID-19 (2019 coronavirus disease), MERS-CoV (middle east respiratory syndrome coronavirus), $95 \% \mathrm{Cl}$ (95\% confidence interval).

Institute of Allergy and Infectious Diseases' phase 3 trial. The approval references the United States' Emergency Use Authorization of the drug to treat severe cases of COVID-19. ${ }^{22}$ Furthermore, Gilead signed a licensing deal recently with 3 Indian companies to manufacture remdesivir. ${ }^{23}$

However, a recent randomized, double-blind, placebocontrolled, multicenter trial published by Wang Y., et al., reported that in this study of adult patients admitted to the hospital for severe COVID-19 (who have pneumonia confirmed by chest imaging, had $\mathrm{SpO}_{2}$ of $94 \%$ or lower on room air or a ratio of arterial oxygen partial pressure to fractional inspired oxygen $\left(\mathrm{PaO}_{2} / \mathrm{FiO}_{2}\right)$ of $300 \mathrm{~mm} \mathrm{Hg}$ or less, or lung infiltrates $>50 \%$ ), remdesivir was not related with statistically significant clinical aids. The patients were admitted to hospital with an interval from symptom onset to enrolment of 12 days or less. In this study, 158 out of 237 patients received I.V remdesivir ( $200 \mathrm{mg}$ on day 1 followed by $100 \mathrm{mg}$ on days 2-10 in single daily infusions) and observed that remdesivir use was not associated with a difference in time to clinical improvement (i.e., decrease in invasive mechanical ventilation, length of oxygen support, hospital length of stay and, days from randomization to discharge, increase in days from randomization to death and a decline of one category, instead of 2 on 6-category scale as discussed by Wang Y., et al.), mortality and viral clearance in patients with severe COVID-19 in comparison with placebo. Clinical improvement rates in remdesivir group were 3\%, 27\% and, $65 \%$ as compared to placebo group of $3 \%, 23 \%$ and, $58 \%$ on day 7, 14 and, 28 respectively. $66 \%$ of 155 remdesivir recipients exhibited adverse events versus $64 \%$ of 78 placebo recipients. The most common adverse events in the remdesivir group were constipation (14\%), hypoalbuminemia (13\%), hypokalemia (12\%), anemia $(12 \%)$, thrombocytopenia (10\%), and increased total bilirubin $(10 \%)$. Weaknesses of this study were deficient power to identify expected differences in clinical outcomes, the commencement of treatment quite late in COVID-19, and the lack of data on infectious virus recovery or on probable emergence of reduced susceptibility to remdesivir. Although, this trial did not achieve the programed sample size due to the outbreak of COVID-19 was taken under control in China. ${ }^{19}$ However, the numerical decline in time to clinical improvement in those treated earlier needs verification in larger studies.

Furthermore, another double-blind, randomized, placebocontrolled trial of I.V remdesivir in adults hospitalized with COVID-19 concluded that remdesivir was superior to placebo in shortening the time to recovery (defined as the first day, during the 28 days after enrollment, on which a patient satisfied categories 1, 2, or 3 on the eight-category ordinal scale discussed by Beigel JH., et al.). ${ }^{20}$ In this study, 538 patients were assigned to remdesivir treatment and 521 to placebo. $88.7 \%$ of patients had the severe disease (hospitalized COVID-19 cases with evidence of lower respiratory tract infection) at enrollment. The median number of days between symptom onset and randomization was 9. The results indicated that remdesivir-treated patients had a median recovery time of 11 days versus 15 days in placebo group. $21.1 \%$ of patients experienced serious adverse effects who received remdesivir while this percentage was $27 \%$ in placebo group. The most common adverse events in the remdesivir group were anemia $(7.9 \%)$, acute kidney injury (7.4\%), pyrexia (5.0\%), hyperglycemia (4.1\%), and increased aminotransferase levels $(4.1 \%)$. Moreover, the estimates of mortality by 14 days were $7.1 \%$ with remdesivir and $11.9 \%$ with placebo. ${ }^{20}$ Though, these findings favors the use of remdesivir for hospitalized COVID-19 patients and who require supplemental oxygen therapy. However, given a high incidence of adverse events and mortality in spite of the use of remdesivir, it is obvious that antiviral drug alone for treating COVID-19 is not likely to be sufficient.

Therefore, for the enhanced safety of patients with COVID-19, further clinical trials and large randomized controlled studies are needed ${ }^{4}$ to validate the effective role, safety profile, and adverse events of remdesivir along with other therapeutic approaches and future strategies. However, any approvals for the usage of a new drug, certainly, needs additional clinical testing, followed by the approval of widespread 
use by the pertinent regulatory authority for medical treatment. To accomplish this, the concentration of the studies may important to be on the direct human clinical approaches in maximum quantity along with in vitro studies to approve the efficacy as well as safety of all pharmacological treatments with remdesivir.

\section{ORCID iDs}

Muhammad Naveed (D) https://orcid.org/0000-0003-4316-8877

Zakir Khan (D) https://orcid.org/0000-0003-1365-548X

\section{References}

1. Huang C, Wang Y, Li X, et al. Clinical features of patients infected with 2019 novel coronavirus in Wuhan, China. The Lancet. 2020;395(10223):497-506.

2. Naveed M, Uddin S, Abdullah KS, et al. Various evidence-based hypothetical and experimental treatment approaches and their effectiveness against COVID-19 worldwide: a comprehensive literature review. EMJO. 2020;4:265-285.

3. Lu H. Drug treatment options for the 2019-new coronavirus (2019-nCoV). Biosci Trends. 2020;14(1):69-71.

4. Khan Z, Karataş Y, Rahman H.Anti COVID-19 drugs: need for more clinical evidence and global action. Adv Ther. 2020:37(6): 2575-2579.

5. Dong $\mathrm{L}, \mathrm{Hu} \mathrm{S}, \mathrm{Gao}$ J. Discovering drugs to treat coronavirus disease 2019 (COVID-19). Drug Discov Ther. 2020;14(1):58-60.

6. Ferner RE, Aronson JK. Remdesivir in Covid-19. BMJP Group. 2020:369:m1610.

7. Clinicaltrials.gov. Remdesivir | Covid-19. 2020.

8. Gordon CJ, Tchesnokov EP, Feng JY, et al. The antiviral compound remdesivir potently inhibits RNA-dependent RNA polymerase from Middle East respiratory syndrome coronavirus. J Biol Chem. 2020;295(15):4773-4779.

9. Elfiky AA. Anti-HCV, nucleotide inhibitors, repurposing against COVID-19. Life Sci. 2020;248:117477.

10. Sheahan TP, Sims AC, Graham RL, et al. Broad-spectrum antiviral GS-5734 inhibits both epidemic and zoonotic coronaviruses. Sci Transl Med. 2017;9(393):eaa13653.
11. Harrison C. Coronavirus puts drug repurposing on the fast track. Nat Biotechnol. 2020;38(4):379-381.

12. (EMA) EMA. Compassionate Use Opinions. European Medicine Agency; 2020.

13. Wang M, Cao R, Zhang L, et al. Remdesivir and chloroquine effectively inhibit the recently emerged novel coronavirus (2019-nCoV) in vitro. Cell Res. 2020;30(3):269-271.

14. Agostini ML, Andres EL, Sims AC, et al. Coronavirus susceptibility to the antiviral remdesivir (GS-5734) is mediated by the viral polymerase and the proofreading exoribonuclease. MBio. 2018;9(2):e00221-18.

15. Li G, De Clercq E. Therapeutic Options for the 2019 Novel Coronavirus (2019-nCoV). Nature Publishing Group; 2020.

16. de Wit E, Feldmann F, Cronin J, et al. Prophylactic and therapeutic remdesivir (GS-5734) treatment in the rhesus macaque model of MERS-CoV infection. Proc Nat Acad Sci USA. 2020;117(12): 6771-6776.

17. Holshue ML, DeBolt C, Lindquist S, et al. First case of 2019 novel coronavirus in the United States. $N$ Engl J Med. 2020; 382(10):929-936.

18. Grein J, Ohmagari N, Shin D, et al. Compassionate use of remdesivir for patients with severe Covid-19. N Engl J Med. 2020; 382(24):2327-2336.

19. Wang Y, Zhang D, Du G, et al. Remdesivir in adults with severe COVID-19: a randomised, double-blind, placebo-controlled, multicentre trial. Lancet. 2020;395(10326):1569-1578.

20. Beigel JH, Tomashek KM, Dodd LE. Remdesivir for the treatment of Covid-19-preliminary report. N Engl J Med. 2020; 383(10):994.

21. Steve Holland DB. U.S. emergency approval broadens use of Gilead's COVID-19 drug remdesivir. US News \& World Report. May 2020.

22. Sternlicht A. Japan approves remdesivir for use On Severe COVID-19 Patients. Forbes. May 2020.

23. Rajagopal D. Gilead sign licensing deal for remdesivir with three Indian companies. The Economic Times. May 2020. 